\title{
APLICACIONES DE MATERIALES MESOPOROSOS EN LA INGENIERÍA DEL TEJIDO ÓSEO
}

\author{
APPLICATIONS OF MESOPOROUS MATERIALS \\ FOR TISSUE ENGINEERING
}

\section{Leglys Contreras-Vargas ${ }^{1}$, Karem Noris-Suarez ${ }^{1}$ \\ \& Gema González ${ }^{2,3 *}$}

Recibido: 1 de octubre 2019 / Aceptado: 12 de noviembre 2020

DOI: DOI 10.26807/ia.v9i1.126

Palabras claves: Ingeniería de tejidos, materiales mesoporosos, regeneración ósea

Keywords: Tissue Engineering, mesoporous materials, bone regeneration, tissue engineering

\section{RESUMEN}

La ingeniería de tejidos se define como un área multidisciplinaria e interdisciplinaria cuya función principal es proponer soluciones novedosas que promuevan la regeneración o reemplazo de tejidos dañados. Las patologías óseas han sido tratadas tradicionalmente con implantes de tejido donado (injertos, aloin-

1 Universidad Simón Bolívar, Laboratorio Bioingeniería de Tejidos, Departamento de Biología Celular. Caracas, Venezuela. (leglyscv@gmail.com; karem.noris@gmail.com)

2 Centro de Ingeniería de Materiales y Nanotecnología. Instituto Venezolano de Investigaciones Científicas (IVIC)

3 Universidad Yachay Tech, Escuela de Ciencias Físicas y Nanotecnología, Urcuquí, Ecuador. ("correspondencia: ggonzalez@yachaytech.edu.ec) 
jertos o xenoinjertos), sin embargo, dichos tratamientos han presentado limitaciones que han promovido el desarrollo y evolución de nuevas estrategias, como es el desarrollo de la ingeniería de tejidos y de biomateriales que puedan fungir como andamios y/o matrices que puedan funcionar como liberadores de drogas. En este sentido, los materiales mesoporosos se proponen como biomateriales bioactivos, cuya característica resaltante es la gran área superficial que presentan debido a sus canales de dimensiones nanométricas. En los últimos años se ha tenido un especial interés en la sílice mesoporosa, ya que es capaz de adsorber una alta cantidad de fármaco y proporcionar una liberación controlada del mismo; también ha resultado ser estimuladora de la osteogénesis aplicándose con grandes expectativas en la regeneración ósea. El presente artículo hace una revisión de las aplicaciones de los materiales mesoporosos a base de sílice en la ingeniería del tejido óseo, tomando en cuenta la importancia del tamaño del poro y biocompatibilidad en el momento de emplearlos como sistemas de adhesión de células y liberación de fármacos.

\section{ABSTRACT}

Tissue engineering is defined as a multidisciplinary and interdisciplinary area whose main function is to propose innovative solutions that promote regeneration or replacement of damaged tissues. Bone's pathologies have traditionally been treated with donor tissue implants (grafts, allografts or xenografts), however these treatments have limitations which have promoted the development and evolution of new strategies, in tissue engineering and biomaterials, which can serve as scaffolds and/or matrix that can function as drug-delivery system. Thus, mesoporous materials are proposed as bioactive biomaterials, whose outstanding feature is the large surface area, due to their channels of nanometric scale. In recent years, mesoporous silica has had a special interest due it has a higher drug load and provides a controlled release. Additionally, also have proved stimulating osteogenesis with high expectations in bone regeneration. This article reviews the applications of mesoporous materials based on silica in bone tissue engineering, taking into account the importance of pore size and biocompatibility on cell adhesion and drug delivery. 


\section{INTRODUCCIÓN}

En la ingeniería de tejidos se busca estimular los mecanismos de regeneración ya presentes de forma natural en el organismo que, además, permitan optimizar la fabricación o diseño de nuevo tejido vivo funcional mediante un soporte (natural, sintético o mezclas de ambos) (Muschler et. al., 2004). En los últimos años se han observado avances importantes en el área de la regeneración ósea, pues se han desarrollado estrategias que permiten trasplantar hueso, cartílago, tendón, ligamento, disco vertebral, entre otros. Uno de estos progresos ha sido el desarrollo de biomateriales, definidos por la Second Consensus Conference on definitions in Biomaterials del Reino Unido en 1992, como materiales diseñados para actuar interfacialmente con sistemas biológicos con el fin de evaluar, tratar, aumentar o sustituir algún tejido, órgano o función del cuerpo (Williams, 1987).

El campo de los biomateriales ha tenido un desarrollo importante, debido a que se ha pasado de utilizar materiales inertes hasta el desarrollo y fabricación de materiales bioactivos y biodegradables, lo que ha Ilevado a una tercera generación de biomateriales, cuyo fin es lograr la regeneración. Para Manzano y Vallet-Regí (2012), los materiales de tercera generación están diseñados considerando que van a estar en contacto con tejidos vivos y que las propiedades de superficie de dichos materiales tales como su topografía, su carga superficial y todos los aspectos relacionados con la química de sus superficies son fundamentales para obtener una respuesta satisfactoria al estar en contacto con el tejido vivo. Por tanto, la evolución en estos materiales ha llevado a una funcionalización adecuada de sus superficies para facilitar la adhesión, proliferación y diferenciación celular en condiciones óptimas.

Los materiales más estudiados para ser aplicados en la regeneración ósea son los polímeros sintéticos (ácido poliláctico, ácido poliglicólico, etc.), matrices naturales como el colágeno, los cementos y las cerámicas. Entre estas últimas presentan especial interés las cerámicas de fosfatos de calcio (por su similitud con la compo- 
sición mineral del hueso) y los vidrios bioactivos (Abarrategui, 2008). Sin embargo, en la regeneración ósea se requiere de piezas que se ajusten al defecto, lo cual ha limitado el empleo de materiales cerámicos, además los biomateriales a utilizar deben presentar buena porosidad que favorezca su colonización por los osteoblastos y permita la angiogénesis (Hutchmacher, 2000; Steven et. al., 2005). Los materiales mesoporosos a base de sílice e hidroxiapatita cumplen con este requisito de porosidad y elevada área superficial, por lo que han tenido diversas aplicacio- nes en el área de tejido óseo y, además de generar gran interés, han cobrado cada vez mayor importancia por las grandes potencialidades que presentan estos materiales.

En tal sentido, el presente artículo tiene como objetivo revisar el uso potencial de materiales mesoporosos de sílice en la ingeniería de tejido óseo, tomando en cuenta la importancia del tamaño de los poros y la biocompatibilidad en el momento de su uso como sistemas de unión y administración de fármacos.

\section{RESULTADOS}

\section{Materiales mesoporosos empleados en la bioingenieria del tejido óseo}

Para comprender cómo los biomateriales podrían reparar o regenerar eficazmente tejido óseo, es necesario entender dicho proceso de formación, tomando en cuenta el potencial de reparación del tejido óseo.

El hueso es un tejido complejo y dinámico que se somete a la renovación y reparación durante toda la vida como resultado de la remodelación ósea. Cuando se produce un trauma, la respuesta del tejido es la regeneración. Esto debido a que el hueso tiene la capacidad de renovarse totalmente tras una lesión (Stewart, 2015). La formación del tejido óseo se da a través de dos procesos básicos: la osificación intramembranosa y la osificación endocondral. La forma más intuitiva de formación ósea es la osificación intramembranosa, es la diferenciación de las cé- 
lulas progenitoras a osteoblastos que producen y depositan directamente el tejido óseo (Stewart, 2015). Este proceso toma lugar típicamente en la periferia del hematoma. El tejido óseo recién formado contribuye a rellenar el defecto y a proveer estabilidad mecánica limitada. El segundo proceso por el cual nuestro cuerpo es capaz de formar hueso es el de la osificación endocondral, en el cual el hueso se forma a través de un tejido cartilaginoso intermedio distinto. Este mecanismo es empleado durante el desarrollo embrionario, en el crecimiento de huesos largos y en las fracturas donde el proceso de regeneración rápidamente forma callos que luego sufrirán la osificación. Esto activa la maquinaria de señalización que permite a las células hacer frente a entornos hipóxicos (Leijten et al., 2014). La tasa, cantidad y calidad de las diversas etapas de la curación ósea están muy influenciadas por una gran cantidad de factores, que incluyen la edad y la salud de los pacientes, el tipo de hueso, la ubicación y el tipo de fractura, la movilidad del sitio de la fractura, suministro de sangre disponible e infección en o cerca del sitio de fractura (Leijten et al., 2015).
La regeneración del tejido óseo sigue siendo un desafío importante en el campo de la cirugía ortopédica y maxilofacial. Los defectos óseos producidos por trauma, tumores, enfermedades infecciosas, trastornos bioquímicos, trastornos congénitos o desarrollo esquelético anormal son las principales causas de discapacidad funcional y trauma estético y psicológico para los pacientes. Uno de los objetivos del tratamiento de un defecto óseo es restaurar la morfología y la función normal de la estructura afectada. Se han desarrollado técnicas quirúrgicas específicas como la osteogénesis por distracción, la implantación de biomateriales (sustitutos óseos) y los implantes de injerto óseo para alcanzar la regeneración ósea (Leijten et al., 2015).

Sin embargo, se siguen presentando limitantes tanto con estas técnicas como con el empleo de cerámicas, lo cual ha llevado a desarrollar biomateriales que puedan fungir como andamios y/o matrices que puedan funcionar como rellenos junto a sustitutos óseos o como liberadores de fármacos, siendo las sílices mesoporosas uno de los biomateriales de mayor interés por sus propiedades 
texturales. De acuerdo con la definición de IUPAC, los materiales mesoporosos son aquellos que presentan un tamaño del poro de 2 a $50 \mathrm{~nm}$, pudiendo tener una mesoestructura desordenada u ordenada (Rouquerol et. al., 1994).

Desde los años noventa se ha reconocido a la familia de materiales mesoestructurados de base silícea, las nanopartículas mesoporosas de sílice (MSNs) se sintetizaron por primera vez en Japón (Yanagisawa et al., 1990). Posteriormente, en 1992 de manera independiente la compañía Mobil Oil Co sintetizó y describió el MCM-41 (por sus siglas en inglés, Mobil Composition of Matter), Ilamado Material Cristalino Mobil (Beck et al., 1992); desde entonces se han sintetizado una gran variedad de estructuras de materiales meosoporosos, en 1998 Zhao et al. (1998) de la Universidad de California (Santa Barbara) desarrollaron nuevos materiales Ilamados SBA (Santa Barbara Acids), en los que se incrementó el tamaño de poro y la estabilidad. El material SBA posee numerosas aplicaciones como catalizadores, soportes, adsorbentes (Thielemann et al., 2011) y como biomaterial de gran interés para el desarrollo de terapias para tratar afecciones óseas.

Los materiales mesoporosos ordenados muestran disposiciones de canales y cavidades con distribución periódica y paredes amorfas construidas a partir de unidades de $\mathrm{SiO}_{2}$ (Ying et. al., 1999). Presentan porosidad ordenada y homogénea con tamaños variables del poro $(2-50 \mathrm{~nm})$, excelentes propiedades texturales con un alta área de superficie (ca. $1000 \mathrm{~m}^{2} / \mathrm{g}$ ), volumen grande de poros (aproximadamente $1 \mathrm{~cm}^{3} / \mathrm{g}$ ), y se puede funcionalizar su superficie con diversos grupos orgánicos (Hoffmann et al., 2006; Sousa et al., 2008). Además, el tamaño de los canales puede ser adaptado mediante la variación del procedimiento de síntesis (Al-Kady et al., 2011).

Los materiales mesoporosos ordenados de sílice han despertado gran interés en el área biomédica debido a sus propiedades de almacenar moléculas de diferentes características, lo que los ha convertido en excelentes sistemas a ser empleados para la liberación de fármacos; también tienen la capacidad de unirse al hueso a través del desarrollo de nanoapati- 
tas similares a las biológicas, las cuales se forman al estar en contacto con fluidos fisiológicos (Colilla y ValletRegí, 2011). Para González et al. (2017) la bioactividad de estos materiales permite una alta afinidad $y$ unión al tejido óseo, lo cual proporciona soluciones alternativas para ciertas patologías óseas, sobre todo para aquellas que requieren tratamiento local. Además, se ha demostrado que los iones de silicio (Si) liberados desde MSN promueven la formación de nódulos de mineralización, síntesis de COLA1 y la expresión de genes osteogénicos (Han et al., 2013; Sun et al., 2009).

Las paredes de los poros de MSNs tienen una alta densidad de grupos silanoles en la superficie que pueden reaccionar frente a moléculas huésped apropiadas (Sousa et al., 2008). Dichos materiales, tienen la ventaja de ser biocompatibles y bioactivos, por lo que han adquirido diversas aplicaciones, tales como: liberación controlada de fármacos (Vivero-Escoto et al., 2009; Shi et al., 2009; Ferreira et al., 2018; Gema et al., 2017; Zanjanizadeh et al., 2018), regeneración de tejido óseo (Su et al., 2012; Lozano et al., 2012; Yachao et al.,
2018) o aplicados para la liberación de drogas en células cancerosas (Wang et al., 2011; Su et al.,2019).

Los MSNs pueden adoptar tres tipos de estructuras, dependiendo del sistema de síntesis seleccionado: 1.arreglo hexagonal de cilindros, 2.- un sistema bi-continuo de poros y 3.- un sistema de laminillas intercaladas.

\section{Materiales de sílice mesoporosas como liberadores de fármacos}

Se ha demostrado que las sílices con recubrimientos de fosfatos de calcio pueden ser osteoinductivas en estudios in vitro con osteoblastos humanos (Phan et al., 2003). Estos materiales se encuentran en ensayos clínicos para ser aplicados en el tratamiento de defectos óseos, tales como osteoporosis, fracturas, osteomielitis, entre otras.

De acuerdo con las propiedades de estos materiales, han sido diversos los estudios que han empleado sílice, bien como recubrimientos o como nanopartículas (Chanes-Cuevas et al., 2018; Chen et al., 2019; Reza et al., 2019; Eivazzadeh-Keihan et al., 2019; Cui et al., 2018; Jafari et al., 
2019). En este sentido, para poder emplear la sílice mesoporosa se debe previamente evaluar su capacidad de degradación, la citotoxicidad y la biocompatibilidad. Para ello debe tomarse en cuenta que tanto la concentración, el tamaño de partícula, la superficie específica y su funcionalización juegan un papel primordial en su toxicidad (Radu et al., 2004; Colilla y Vallet-Regí, 2011).

Actualmente se han venido desarroIlando estrategias para la incorporación en estas matrices de agentes osteogénicos (fármacos y moléculas bioactivas) que permitan inducir la regeneración del tejido óseo a través de la liberación controlada de factores de crecimiento (Quan et al., 2012), proteínas morfogénicas óseas (Yilgor et al., 2009), alendronato (Nieto et al., 2008), ibuprofeno (Shou-Cang, 2011; González et al., 2013) diversos antibióticos (Shi et al., 2009), entre otros.

La evolución en estas aplicaciones se centra tanto en la liberación localizada en la que el fármaco sólo actúa en el tejido diana donde se necesita, como en el control de la dosificación, de manera que el fármaco se li- bera de forma controlada durante un determinado periodo de tiempo (Nieto, 2011).

\section{Aplicaciones de los materiales meso- porosos en la bioingeniería del tejido óseo}

\section{Importancia de la porosidad para los sistemas de carga y liberación en los materiales mesoporosos}

Las características de superficie y tamaño de los poros de los materiales mesoporosos son de especial importancia al desarrollarlos para el tratamiento de patologías óseas, esto debido a que es posible emplearlos como transportadores de fármacos o agentes osteogénicos para su posterior liberación. El tamaño del poro determina el tamaño de la molécula que se desea liberar, de igual manera es importante para controlar la velocidad de difusión (Fan et al., 2003). Por lo tanto, si el tamaño del poro es más grande que la dimensión de la molécula a cargar, son suficientes para lograr la adsorción; ahora bien, si el poro es menor que la molécula solo se podrá adsorber en la superficie externa del material (Colilla y Vallet-Regí, 2011). En los últimos años, 
los mesoporosos a base de sílice han tenido un mayor uso para sistemas de liberación de medicamentos, entre estos materiales los más importantes han sido: MCM-41 (Simetría hexagonal), SBA-15 (Santa Barbara), MCM50 (simetría laminar), HMS (Sílice Mesoporosa Hexogonal) y TMS (Sílice mesoporosa conteniendo titanio).

En trabajos realizados por Heikkilä et al. (2007) un tamaño de poro grande resultó en una liberación más rápida del fármaco que en sistemas de liberación controlada. En este mismo sentido, Fengyu et al. (2006) al estudiar la liberación de captopril en estructura hexagonal ordenada (2D) (MCM-4112, MCM-4116, SBA-15), verificaron que la cantidad final adsorbida del fármaco depende de la superficie del material mesoporoso. Estos autores investigaron la influencia del tamaño de poro en la cinética de carga y liberación del fármaco, señalando que dicho efecto puede evaluarse solo si la morfología es similar a nivel de microestructura.

No se debe dejar a un lado la influencia que tiene el tamaño del poro al liberar moléculas grandes como proteí- nas que tienen un tamaño similar al poro del biomaterial. Investigaciones han indicado que al cargar proteínas con dimensiones superiores al diámetro de MCM-41 (DP=1,5-3,5 nm), solo pueden adsorberse en la superficie del mismo (Yiu et al., 2005). Así, proteínas con tamaños más pequeños que el diámetro del mesoporoso muestran una mejor adsorción y liberación, por tanto, el tamaño del poro puede cumplir un efecto de tamiz molecular para moléculas grandes y controlar la velocidad de liberación. En lo que se refiere a moléculas grandes empleadas para la regeneración ósea, se pueden mencionar a las proteínas morfogenéticas (BMP) o bien a la albúmina sérica, ésta última ha sido de interés para el área porque puede enlazar diferentes fármacos que luego pueden ser liberados en el lugar de acción farmacológico necesario (Peters, 1996). Por lo general, los mesoporosos SBA-15 son los más empleados para la albúmina sérica y otras moléculas de mayor dimensión.

Para solventar esta limitante del tamaño se puede funcionalizar la MSN, para ello se modifican las propiedades fisicoquímicas del material incorporando componentes orgáni- 
cos e inorgánicos en su superficie, estructura o bien atrapados en sus canales (Hoffman et al., 2006). Esta modificación permite optimizar las interacciones del fármaco y el transportador. Los materiales mesoporosos son un soporte interesante para grupos funcionales orgánicos debido a sus propiedades estructurales (Zeid et al., 2012). Para aplicaciones biológicas son empleados con mayor frecuencia grupos amino y carboxilo, esto debido a que permiten una mayor interacción de las moléculas lo cual puede ser empleado para inmovilizar biomoléculas a través de enlaces covalentes (Quianjun y Jianlin, 2011). Sin embargo, la modificación de la superficie del material mesoporoso dependerá del fármaco a cargar y de su aplicación; por ejemplo, se ha demostrado que los MSN modificados con péptidos de arginina-glicinaácido aspártico (RGD) pueden mejorar drásticamente la adhesión celular del hidrogel de alginato en comparación con el hidrogel incorporado en $\mathrm{MSN}-\mathrm{NH}_{2}$ (Kehr et al., 2013). Por lo tanto, resulta una ventaja la funcionalización de las MSN lo que permite el acceso a las propiedades texturales deseadas para aplicarla en la Bioingeniería de Tejido.
En el campo de los biomateriales, la hidroxiapatita (HA) ha sido uno de los más empleados en el área de la regeneración ósea. Ha sido empleada como injerto de hueso, pero por sí sola presenta bajas tasas de reactividad y de integración ósea, sin embargo, si se combina con átomos de sílice esto conlleva a un aumento de los procesos de disolución, incrementando la tasa de incorporación del injerto haciéndolo más osteoinductivo (Porter et al., 2004). Nuestros estudios con SBA-15 y SBA-16 (González et al., 2017) han permitido la formación de una capa de apatita en las paredes de la sílice mesoporosa, lo cual ha sido posible a través de la inmersión de las MSNs en fluido corporal simulado (SBF) por un lapso de una semana. La Figura 1 muestra la formación de nanopartículas de hidroxiapatita interactuando con la estructura mesoporosa de la sílice, junto con un espectro de microanálisis elemental indicando la presencia de $\mathrm{Ca}, \mathrm{P}$ de la hidroxiapatita y $\mathrm{Si}$ componente de la matriz mesoporoso.

La formación de la capa de apatita resulta en la disminución en el área de superficie (65\% SBA-16 y $45 \%$ 
para SBA-15), área mesoporosa y volumen del poro (29\% para SBA-16 y $45 \%$ en SBA-15). El área de microporos se vuelve casi insignificante para ambos materiales, pero el diámetro de poro mostró un aumento importante (36\% para SBA-16/Ha y $62 \%$ para SBA-15/Ha, sugiriendo la formación de nuevos poros irregulares con una nueva estructura de referencia. De igual manera, se determinó el incremento de la bioactividad del SBA-15/HA, comparado con SBA-15 sin recubrimiento de HA. Confirmando de esta forma el papel de la capa de apatita y el potencial de aplicarla en combinación con MSNs.

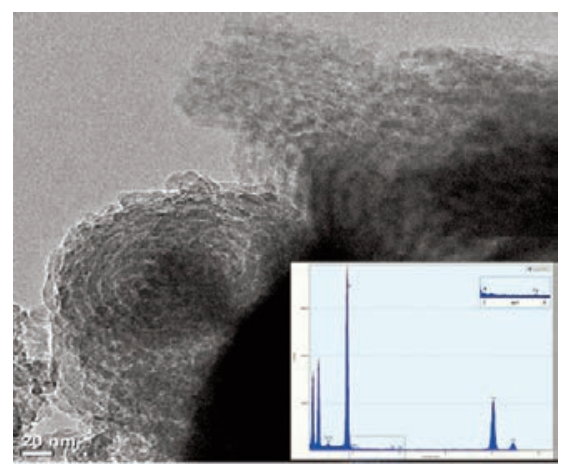

Figura 1. Microscopía de transmisión electrónica de nanocristales de hidroxiapatita SBA-15 sobre la superficie de una pared mesoporosa. Adentro: EDS microanálisis.

\section{Comportamiento in vitro e in vivo de sílice mesoporosa (MS)}

Al trabajar con biomateriales es indispensable evaluar su biocompatibilidad y la capacidad de reabsorción, ellos permanecen en contacto con los tejidos vivos. De acuerdo con esto, al estudiar su comportamiento in vitro no deben producirse reacciones inadecuadas en la interacción tejido-material. Así, al evaluar la viabilidad de sílices mesoporosas para la regeneración de tejido óseo, el primer paso debe consistir en la realización de ensayos con cultivos de células osteoblásticas. En este sentido, García et al. (2009) realizaron ensayos con osteoblastos empleando diferentes materiales mesoporosos sintetizados en un sistema binario con $\mathrm{SiO}_{2}-\mathrm{P}_{2} \mathrm{O}_{5}$ con diferentes concentraciones de fósforo. Para evaluar la citotoxicidad y viabilidad celular, se determinó la actividad de la enzima lactato deshidrogenasa (LDH) en los medios de cultivos a los 2 días de ensayos y la proliferación celular se llevó a cabo utilizando MTT a los 2 y 6 días de cultivo. Los resultados indican el menor daño celular (citólisis) para los ensayos en $\mathrm{Si}_{95} \mathrm{P}_{5}$, independientemente de la concentración del mate- 
rial utilizado en comparación con las muestras que se encontraban en sílice puro $\left(\mathrm{Si}_{100}\right)$ y $\mathrm{Si}_{97,5} \mathrm{P}_{2,5}$ que presentan diferencias no significativas. Se obtuvo un aumento de LDH con el incremento en la concentración de materiales. Esto se debe a que la cantidad de lixiviado de sílice es mayor cuando aumenta la concentración y por lo tanto el número de partículas por célula también se incrementa, lo que podría producir un mayor grado de toxicidad. La mayor viabilidad celular se presentó en los ensayos con $\mathrm{SiO}_{2}-\mathrm{P}_{2} \mathrm{O}_{5}$.

Actualmente se ha empleado la combinación de sílices mesoporosas con otros componentes, así como se han venido desarrollando materiales por el método sol-gel los cuales presentan numerosas aplicaciones, algunas incluyen la incorporación de especies biológicamente activas en estas matrices evidenciando su bioactividad. Nieto et al. (2009) desarrollaron un procedimiento sol-gel en dos etapas para la obtención de materiales porosos de sílice que permitan la encapsulación de células vivas (fibroblastos y células epiteliales) en su interior durante la síntesis, así como el posterior estudio de su viabilidad a corto plazo. El proceso sol-gel se llevó a cabo en dos etapas, primero una hidrólisis ácida partiendo de tetraetilortosilicato (TEOS) como precursor de sílice, seguido de una etapa de condensación-gelificación en medio neutro. La incorporación de las células al sol neutro, estimula su gelificación inmediata quedando atrapadas en la matriz silícea, utilizando moldes cilíndricos para conformarlos como piezas monolíticas. Se comprobó la bioactividad y no toxicidad del mismo, demostrando que las células pueden sobrevivir a las condiciones de encapsulación dentro de geles de sílice en estado húmedo. Dicha agrupación celular ha demostrado ser un método eficaz para mejorar la fijación celular, ya que protege a las células encapsuladas y mejora la viabilidad celular.

De acuerdo con esta investigación, las matrices silíceas sintetizadas vía sol-gel pueden ser consideradas como buenas candidatas en aplicaciones en biomedicina para el diseño de andamios y para el cultivo de tejidos.

Los estudios in vitro son un gran avance para llegar a experimentar in vivo, ya que son condiciones diferen- 
tes en las que pueden variar las respuestas celulares, así como se obtienen mejores respuestas en lo que se refiere a rechazo del implante o material por parte de organismo. En este sentido, las investigaciones de Hudson et al. (2008) demostraron la biocompatibilidad in vivo de tres tipos de materiales mesoporosos: MCM41, SBA-15 y MCF observándose que la toxicidad en las células mesoteliales y mioblastos incrementa con la concentración de las partículas, encontrándose altas cantidades en las células mesoteliales. Asimismo, al inyectar los materiales por la vía subcutánea en el nervio ciático de las ratas, se obtuvo a nivel histológico una buena biocompatibilidad en todos los tiempos tratados. Sin embargo, a pesar de este resultado las inyecciones intraperitoneal e intravenosa dan como resultado la muerte de los animales, las razones no están claras, pero pueden atribuirse a una trombosis pulmonar. Se conoce que la sílice amorfa no resulta tóxica para el organismo, por el contrario, se ha comprobado que es biocompatible y degradable en el tejido vivo. No obstante, siempre en su composición puede encontrarse un porcentaje de sílice cristalina, que es la que puede conducir la fibrosis pulmonar (Martín, 2007). Este tipo de investigación aporta información con respecto a la importancia de la ruta de administración en el organismo.

Otros estudios in vivo como los de Lozano et al. (2012) han demostrado la capacidad del SBA-15 funcionalizado o no con grupos C8 (C8-SBA15), cargados con la PTHrP (péptido 107-111 u osteostatina) para inducir osteogénesis en un modelo de defecto cavitario en el fémur del conejo, obteniendo a las 4 semanas de la lesión, osteointegración con presencia de tejido conectivo y osteoide. Así, el SBA-15 es biocompatible y al cargarlo con algún agente osteogénico, tal como la osteostatina, se obtiene un importante efecto modulador de la formación ósea.

Los estudios con MSNs han demostrado baja citotoxicidad sin efectos adversos en varios tipos celulares (Huang et al., 2008; Luo et al., 2015; Ren et al., 2015). En cultivos de osteoblastos hemos obtenido una excelente biocompatibilidad al estar expuestos por 14 días con SBA-15 y SBA-15/HA (González et al., 2017). Investigaciones han reportado resul- 
tados positivos trabajando a concentraciones que van desde los $10 \mu \mathrm{g} / \mathrm{mL}$ hasta los $500 \mu \mathrm{g} / \mathrm{mL}$ (Huang et al., 2008; Luo et al., 2015), sin embargo, Gonzalez et al., (2017) emplearon una concentración de 0,6 mg/mL la cual mantuvo la viabilidad celular. Por encima de $0,6 \mathrm{mg} / \mathrm{mL}$ comienza a disminuir la proliferación celular.

\section{Materiales de sílice mesoporosas como agentes liberadores de fárma- cos para afecciones óseas}

La formación del hueso es un proceso complejo y dinámico que implica la participación de diversos factores de crecimiento que llegan a la matriz ósea, donde quedan retenidos para cumplir con su función biológica a través de su liberación y presentación a células efectoras. Así, en el hueso encontramos naturalmente factores de crecimiento tales como: proteínas morfogenéticas óseas (BMPs), factor de crecimiento de fibroblastos (FGF), el factor de crecimiento insulínico (IGF), factor de crecimiento endotelial vascular (VEGF) y factores de crecimiento transformadores $\alpha$ y $\beta$ (TGF- $\alpha$ y $\beta$ ). Estas moléculas regulan las actividades celulares, por lo que se les ha to- mado en cuenta para su aplicación como agentes bioactivos en la reparación del tejido óseo, en el control del crecimiento y en la diferenciación celular (Lee et al., 2002).

\section{Proteínas Morfogenéticas Óseas (BMPs)}

Las BMPs son citoquinas multifuncionales que se encuentran en la familia de los factores de crecimiento transformante beta (TGF- $\beta$ ). Se ha demostrado la capacidad osteoinductora de estas proteínas (BMP-2, BMP-4, BMP-6, BMP-7, BMP-8 у BMP-9) (Bozal, 2006; Boyle y Lacey, 2003). Se ha determinado que están implicadas en diversas funciones biológicas en lo que respecta a curación de fracturas, así como en el reclutamiento, estimulación y proliferación de células mesenquimales y su diferenciación a osteoblastos (Wozney et al., 1988; Erlebacher et al., 1998).

Luo et al., (2015) consideran que la incorporación de proteínas derivadas de BMP-7 mejora notablemente la proliferación y diferenciación osteogénica en cultivos de células MG-63. Esto lo comprobaron al cargar un péptido derivado de BMP-7 en MSNs 
donde obtienen una liberación sostenida del péptido en 6 días in vitro, la carga del péptido en la sílice mesoporosa puede deberse a los puentes de hidrógeno o a los enlaces de amina iónica electrostática de largo alcance $\left(\mathrm{Si}-\mathrm{O}^{-}-{ }^{+} \mathrm{H}_{3} \mathrm{~N}\right)$ formado entre el grupo silanol en la MSN y el N-terminal de las cadenas de los aminoácidos Gln, Thr o el grupo amino de la primera Ala. Este sistema estimula a partir del día 5 la proliferación celular en concentraciones de $500 \mu \mathrm{g} / \mathrm{mL}$ de péptido-MSNs, exhibiendo, además, una excelente biocompatibilidad. Por tanto, concentraciones por encima de 100 g/mL de péptido-MSNs liberan suficientes moléculas de la proteína como para desencadenar la estimulación de la osteogénesis.

\section{Factor de crecimiento insulínico (IGF)}

El factor de crecimiento IGF ha sido de gran interés en lo que respecta a la regeneración ósea, ya que tienen la capacidad de estimular la proliferación y migración de diferentes células óseas indispensables para la curación del hueso. Se sintetiza en diferentes tejidos y tienen sus efectos en forma endocrina, paracrina y au- tocrina (Pollak, 2012). IGF-I e IGF-II son los más importantes en el metabolismo óseo, en el crecimiento del esqueleto y mantenimiento de la masa ósea. Estudios Ilevados a cabo por Quan et al., (2012) demostraron que vidrios mesoporosos bioactivos tienen in vitro la capacidad de promover la diferenciación de osteoblastos al inducir la expresión de IGF-II y obtener una elevación de la actividad de fosfatasa alcalina.

Mendes et al., (2013) fabricaron una MSN a través de precursores inorgánicos, fosfato cálcico y surfactante no iónico, para evaluar la carga y liberación del péptido de crecimiento osteo génico (OGP) a diferentes concentraciones para su aplicación en la regeneración ósea. Se ha demostrado in vivo que la OGP regula la expresión de factores de crecimiento como el factor de crecimiento transformante (TGF), factor de crecimiento fibroblástico (FGF-2) y el factor de crecimiento insulínico tipo 1 (IGF-1) (Brager et al., 2000) y estimula la proliferación de osteoblastos y activación de la fosfatasa alcalina (Vanella et al., 2010). Para Ilevar a cabo este trabajo se fabricaron dos tipos de sílice mesoporosa, una pura $(\mathrm{SiO})$ y 
otra recubierta con hidroxiapatita (SiCaP). Las muestras fueron obtenidas en forma de discos de $7 \mathrm{~mm}$ de diámetro y $1 \mathrm{~mm}$ de espesor. Los resultados indicaron una relación directamente proporcional a la concentración de la partícula. En ambas matrices se obtuvo una efectiva liberación del péptido, la cual pudo ser beneficiada por la interacción entre la biomolécula y la sílice por los puentes de hidrógeno y la afinidad al N-terminal del OGP con las cadenas de silanol presentes en la muestra. Sin embargo, el sistema SiO-OGP presenta una rápida tasa de liberación para las primeras 9 horas liberando cerca del $60 \%$ del péptido incorporado, luego de 68 h, cerca del $85 \%$ es liberado y este valor se mantiene constante. Si algo se debe destacar es que esta liberación inicial explosiva es una de las variables a controlar para evitar la toxicidad del material.

\section{Factor de crecimiento fibroblástico (FGF)}

Los FGF son considerados potentes reguladores del crecimiento de células y cicatrización de heridas, constituidos por más de 25 tipos. Estos factores actúan en la fase temprana de la osteogénesis (Kempen et al., 2010). De igual manera, pueden mejorar la bioactividad de materiales, lo cual ha sido demostrado por ShinHee et al. (2011) quienes emplearon capas de recubrimiento con xerogel de sílice y quitosano como medio de liberación de FGF y para mejorar la bioactividad del titanio. Los resultados indicaron una mejor adhesión y expansión de células osteoblásticas obteniendo una alta actividad de fosfatasa alcalina a los diez días de tratamiento.

A la hora de trabajar con FGF es importante tomar en cuenta que dicho factor es degradado rápidamente, de igual manera debe tenerse precaución en los sistemas de liberación, ya que altas dosis pueden llevar a una inhibición de los procesos de osteogénesis, tal como lo indican Quarto y Longaker (2006) al obtener inhibición in vitro de la osteogénesis en células estromales al estar expuestas a FGF-2.

\section{Bifosfonatos}

La osteoporosis se conoce como una enfermedad caracterizada por la pérdida de masa ósea junto con un de- 
terioro de la microarquitectura que incrementa la fragilidad del hueso y le predispone a sufrir fracturas (Raisz y Rodan, 2003). La bioingeniería de tejidos ha venido incorporando bifosfonatos en sílices mesoporosas en búsquedas de un mejor tratamiento que permita mayor absorción y eficiencia del medicamento para tratar la perdida de densidad ósea. Así, la liberación del fármaco se controla generalmente por difusión debido a que la matriz de la sílice permanece sin cambios durante la liberación, por lo que dicha cinética puede describirse con las leyes de Fick (Zhao et al., 2009; Tang et al., 2006). Sin embargo, cuando las interacciones entre las moléculas del fármaco y la pared de la matriz son más fuertes y específicas, la liberación no puede controlarse por esta vía, sino más bien por la estabilidad del complejo entre los grupos funcionales en la pared del poro y los de la droga. Este último tipo de regulación es común para la liberación del fármaco desde superficies de MS funcionalizadas (Ukmar y Planinsek, 2010). En este sentido, Nieto et al. (2008) diseñaron un sistema de liberación de alendronato sódico basado en materiales mesoporosos ordenados de sílice SBA-15 a través del efecto del grado de funcionalización con grupos aminopropilo, a fin de favorecer la adsorción del alendronato sódico. Los resultados indican que el método catalizado induce una carga gradual aumentando la cantidad del fármaco adsorbido a medida que incrementa la cantidad de grupos aminopropilo en la superficie del material mesoporoso, la adsorción es modulable. Dicho comportamiento se debe a la interacción química entre los grupos fosfonato en el alendronato con los grupos silanol de los materiales. Por tanto, la adsorción de la biomolécula está controlada tanto por su accesibilidad a los grupos aminopropilo como por su velocidad de difusión molecular a través de la matriz mesoporosa, determinándose que la velocidad de liberación del fármaco es independiente del grado de funcionalización, sin embargo, la cantidad máxima liberada está relacionada con el número de grupos aminopropilo anclados en la superficie de la sílice. En otras investigaciones han mejorado la liberación controlada del alendronato combinando la sílice mesoporosa con hidroxiapatita (HA), mediadas en microesferas de ácido poli (lácticoco-glicólico) (PLGA) (Shi et al., 2009). 
Se muestran como un prometedor transportador de moléculas bioactivas, el sistema de suministro in situ de AL es factible emplearlo para tratar afecciones óseas, como la osteoporosis.

En este mismo criterio de investigaciones, Manzano et al. (2011), establecieron una herramienta útil para el diseño de sistemas implantables de liberación de fármacos, que en un futuro permitirá predecir con anticipación la cinética de liberación de biomoléculas. Dichos investigadores modelaron un sistema de liberación de zoledronato empleando la matriz SBA-15. Los resultados indican una carga exitosa del fármaco, confirmándose su presencia en la matriz por la formación de enlaces $\mathrm{C}-\mathrm{H}$, el cual es un indicativo de la presencia de la cadena alquilo del fármaco. También pudo encontrarse la presencia de imidazol. Otro aspecto importante en dicho modelo es el mantenimiento de la estructura de la matriz mesoporosa luego de la carga. En cuanto a la liberación del fármaco, se obtuvo estallido de efecto inicial, seguido de una liberación más estacionaria. Uno de los aspectos a tomar en cuenta a la hora de cargar fármacos de este tipo es que las partículas de los materiales adsorbidos se forman como comprimidos (discos), así, durante el proceso de compactación, se obtienen pequeños túneles entre las partículas. Estos microporos resultan mayores que los presentes dentro de las partículas mesoporosas, este factor es importante para modelar la cinética de liberación de este tipo de matrices.

\section{Antibióticos}

Otro de los inconvenientes que se presentan con frecuencia en el reIleno del hueso y en los implantes, son las infecciones que por lo general viene acompañadas de un proceso de deterioro del tejido óseo hasta llevarlo a la destrucción (Manzano et al., 2012). Por este motivo se han estudiado sistemas de liberación local de antibióticos que permitan administrar dosis controladas del fármaco para tratar la infección y a la vez se promueva la formación del hueso.

Las bacterias Staphylococcus aureus y Staphylococcus epidermidis son las principales causantes de la mayoría de las infecciones relacionadas con implantes (Wu et. al., 2003). Dichas 
infecciones han sido motivo para el desarrollo de un sistema de implante liberador de fármaco, que consta de un lecho relleno de microesferas mesoporosas del tipo MCM-48 dentro de un depósito de pared porosa de acero inoxidable. Se busca controlar la velocidad de liberación del fármaco para combatir infecciones bacterianas causadas por Staphylococcus aureus cuyo crecimiento fue evaluado mediante la liberación de linezolid, previamente adsorbido sobre las micropartículas de sílice. Se pudo observar luego de 48 horas un bajo crecimiento y adhesión de bacterias a las sílices mesoporosas cargadas con linezolid, corroborando de esta forma el efecto bactericida del fármaco. De igual forma, la resistencia mecánica del acero poroso propuesto como un pasador de elución de fármacos implantable fue probado, encontrándose satisfactorio para el uso clínico (Pérez et al., 2011).

Materiales multifuncionales han sido desarrollados por Shi et al. (2009): se trata de un compuesto producido mediante la hibridación de HMS-HA y PLGA. El antibiótico liberado fue la gentamicina (GS), ya que es el más aplicado en caso de ostiomielitis. El fármaco fue cargado en las partículas HMS-HA y luego incorporados por esferas de PLGA para su posterior liberación controlada. La aplicación de PLGA se hizo con el fin de aumentar la unión de las partículas de HMS-HA en el andamio modificado, además hace que el tiempo de liberación del fármaco sea más prolongado. Este tipo de diseño permite un tiempo de liberación de GS de hasta 12 horas, obteniendo una buena linealidad. Estos andamios combinados de HMS-HA y PLGA muestran propiedades mecánicas similares al hueso esponjoso humano, lo que podría ser un candidato factible para la regeneración del tejido óseo. Además, los andamios de PLGA/HMSHA presentan efectos positivos en el suministro de fármacos y la proliferación celular. De acuerdo con esta evidencia, el desarrollo de andamios bifuncionales ofrece más ventajas para aplicaciones clínicas. 


\section{CONCLUSIONES}

El carácter interdisciplinario y multidisciplinario de la ingeniería de tejido, ha llevado a crear materiales mesoporosos para proporcionar desde el punto de vista estructural, no solo el ambiente necesario de la integridad requerido para la regeneración ósea, sino que se están diseñando con la capacidad de controlar la dosificación y la cinética de liberación del fármaco. Este avance reviste un especial interés en patologías como la osteoporosis, que se espera tratarla a futuro a través de estos nuevos métodos de administración de fármacos. De igual forma, estudios in vivo han sido factibles y positivos hacia el tratamiento de fracturas, convirtiéndose en una esperanza para acelerar los procesos de regeneración ósea.

Así, la aplicación de materiales mesoporosos de sílice como matrices para la adsorción y liberación de fármacos, tales como antibióticos y agentes osteogénicos reúne prometedoras esperanzas para su aplicación en la ingeniería del tejido óseo. Aunque la MSN se encuentra en su etapa de desarrollo, se deben aumentar los estudios in vivo sobre la biodegrabilidad y biocompatibilidad a largo plazo. 


\section{LISTA DE REFERENCIAS}

Abarrategui, A. (2008). Estudio del Quitosano como Biomaterial Portador de rhBMP-2: Desarrollo, Caracterización y Aplicabilidad en Regeneración de Tejido Óseo. Universidad Complutense de Madrid: Tesis Doctoral.

Al-Kady, A., Gaber, M., Mohamed, M., Hussein, E., and Ebeid. (2011). Nanostructureloaded mesoporous silica for controlled release of coumarin derivatives: A novel testing of the hyperthermia effect. European Journal of Pharmaceutics and Biopharmaceutics. 77: 66-74.

Beck, J., Vartuli, J., Roth, W., Leonowicz, M., Kresge, C., Schmitt, K., Chu, C., Olson, D., Sheppard, E., McCullen, S., Higgins, J. and Schlenker, J. (1992). A new family of mesoporous molecular sieves prepared with liquid crystal templates. American Chemical Society. 114: 10834-10843.

Colilla, M and Vallet-Regi, M. (2011). Inorganic and Hybrid Controlled Release Systems Chapter 4. Ordered Mesoporous Silica Materials (1st Ed. 497-514) Universidad Complutense de Madrid, Spain, Elsevier Ltd.

Chanes-Cuevas, O.A., Perez-Soria, A., Cruz-Maya I., Guarino V., Alvarez-Perez M.A., (2018) Macro-, micro- and mesoporous materials for tissue engineering applications AIMS Materials Science, 5(6): 1124-1140.

Chen, L., Zhou, X., He, C. (2019). Mesoporous silica nanoparticles for tissue $\_$engineering applications. Nanomedicine and Nanobiotechnology; e1573, 1-22.

Cui, W., Liu, Q. Q., Yang, L., Wane, K., Sun, T. F., Ji, Y. H., Guo, X. D. (2018). Sustained delivery of BMP-2-related peptide from the true bone ceramics/hollow mesoporous silica nanoparticles scaffold for bone tissue regeneration. ACS Biomaterials Science \& Engineering, 4, 211-221.

Eivazzadeh-Keihan, R., Chenab, K. K., Taheri-Ledari, R., Mosafer, J., Hashemi, S. M., Mokhtarzadeh, A., Hamblin, M. R. (2019). Recent advances in the application of mesoporous silica-based nanomaterials for bone tissue engineering. Materials Science and Engineering: C, 110267. 
Erlebacher, E., Filvaroff, J., Ye, R., Derynck. (1998). Osteoblastic responses to TGF-beta during bone remodelling. Mol. Biol. Cell. 9: 1903-1918.

Fan, C., Yu, F., Gao, J., Lei, B., Tian, L., Wang, Q.,Luo, B., Tu, W., Zhou, D., Zhao. (2003). Cubic mesoporous silica with large controllable entrance sizes and advanced adsorption properties. Angew. Chem. Int. 42: 3146-3150.

Feng, L., Ward, S., Liu, Y., Lu, Y., Xie, B., Yuan et al. (2006). Loss of DMP1 causes rickets and osteomalacia and identifies a role for osteocytes in mineral metabolism. Nat Genet. 38: 1310-1315.

Fengyu Q., Zhu G., Huang, S., Li, S., Sun, J., Zhang, D and Qiu, S. (2006). Controlled release of Captopril by regulating the pore size and morphology of ordered mesoporous silica. Microporous and Mesoporous Materials. 92: 1-9.

Ferreira, G., Faria, J., Gomes, D., Barros, A., Fernandes, R., Soares, A., Takahashi, J., Barros, E. (2018). Mesoporous silica SBA-16/hydroxyapatite-based composite for ciprofloxacin delivery to bacterial bone infection. Journal of Sol-Gel Science and Technology. Vol. 85 (2), pp 369-381.

García, A., Colilla, M., Izquierdo-Barba, I., and Vallet-Regí, M. (2009).Incorporation of phosphorus into mesostructured silicas: a novel approach to reduce the $\mathrm{SiO} 2$ leaching in water. Chem. Mater. 21: 4135-4145.

Gonzalez, G., A. Sagarzazu, T. Zoltan, J. (2013). Infuence of Microstructure in Drug Release Behavior of Silica Nanocapsules. Journal of Drug Delivery. 803585 1-8.

Gonzalez. G, Sagarzazu. A, Cordova. A, Gomes. M, Salas. J, Contreras. L, Noris. K, L. Lascano. (2017). Comparative study of two silica mesoporous materials (SBA-16 and SBA-15) modified with a hydroxyapatite layer for clindamycin controlled delivery. Microporous and Mesoporous Materials. 256, 251-265

Han, P., Wu, C., Xiao, Y. (2013). The effect of silicate ions on proliferation, osteogenic differentiation and cell signalling pathways (WNT and $\mathrm{SHH}$ ) of bone marrow stromal cells Biomater. Sci. 1, 379-392 
Heikkilä, T., Salonen, J., Tuura, J., Hamdyb, M., Mulb, G., Kumarc, N., Salmi, T., Murzin, D., Laitinen, L., Kaukonen, A., Hirvonen, J., Lehto, V. (2007). Mesoporous silica material TUD-1 as a drug delivery system. International Journal of Pharmaceutics. 331:133-138.

Hoffmann, M., Cornelius, J., Morell and M. Fröba. (2006). Silica-based mesoporous organic inorganic hybrid materials. Angew. Chem. Int. 45: 3216-3251.

Huang D-M, Chung T-H, Hung Y, Lu F, Wu S-H, Mou C-Y. (2008). Internalization of mesoporous silica nanoparticles induces transient but not sufficientosteogenic signals in human mesenchymal stem cells. Toxicol Appl Pharm; 231:208-15.

Hudson, S., Padera, R., Langer, R and Kohane, D. (2008). The Biocompatibility of Mesoporous Silicates. Biomaterials. 29(30): 4045-4055.

Hutmacher, D. W. (2000). Polymeric Scaffolds in Tissue Engineering Bone and Cartilage. Biomaterials, 21, 2529-2543.

Jafari, S., Derakhshankhah, H., Alaei, L., Fattahi, A., Varnamkhasti, B. S., \& Saboury, A. A. (2019). Mesoporous silica nanoparticles for therapeutic/diagnostic applications. Biomedicine \& Pharmacotherapy, 109, 1100-1111.

Kempen, D., Creemers, L., Alblas, J., Lu, L., Verbout, A., Yaszemski, M., and Dhert, W. (2010).Growth Factor Interactions in Bone Regeneration. Tissue Engineering: Part B, 16 (6): 551-566.

Kehr, N. S., Prasetyanto, E. A., Benson, K., Ergun, B., Galstyan, A., \& Galla, H. J. (2013). Periodic mesoporous organosilica-based nanocompositehydrogels as three-dimensional scaffolds. Angewandte Chemie-International Edition.52, 1156-1160.

Lee, J., Nam, S., Park, Y., Lee, Y., Seol, Y., Chung, C., Lee, S. (2002). Enhanced bone formation by controlled growth factor delivery from chitosan-based materials. J Control Release. 78(1-3):187-97.

Leijten, N. Georgi, L. Moreira Teixeira, C.A. van Blitterswijk, J.N. Post, M. Karperien. (2014). Metabolic programming of mesenchymal stromal cells by oxygen tension directs chondrogenic cell fate. Proc. Natl. Acad. Sci. U. S. A. 111, 13954-13959. 
Leijten, J., Chai, Y., Papantoniou, I., Geris, L., Schrooten, J and Luyten. (2015). Cell based advanced therapeutic medicinal products for bone repair: Keep it simple? Advanced Drug Delivery Reviews. 84, 30-34

Lozano, D., Manzano, M., Doadrio, J., Salinas, A., Vallet-Regi, M., Gomez-Barrena, E et al. (2012). Osteostatin-loaded bioceramics stimulate osteoblastic growth and differentiation. Acta Biomaterialia. 6, 797-803.

Luo Z, Yi D, Zhang R, Wang M, Bai Y, Zhao Q, Lyu Y,Wei J, Wei S. (2015). Peptide-laden mesoporous silica nanoparticles with promoted bioactivity and osteo-differentiation ability for bone tissue engineering. Colloids and Surfaces B: Biointerfaces, $13173-82$.

Manzano, M., Lamberti, G., Galdi, I., and Vallet-Regí, M. (2011). Anti-Osteoporotic Drug Release from Ordered Mesoporous Bioceramics: Experiments and Modeling. AAPS PharmSciTech.12 (4), 1193-1199.

Manzano, M and Vallet-Regí, M. (2012). Revisiting bioceramics: Bone regenerative and local drug delivery systems. Solid State Chemistry 40, 17-30.

Martin, K. (2007). The chemistry of silica and its potential health benefits. Journal of Nutrition, Health \& Aging. 11(2), 94-98.

Mendes, L., Saska, S., Martines, M and Marchetto. (2013). Nanostructured materials based on mesoporous silica and mesoporous silica/apatite as osteogenic growth peptide carriers. Materials Science and Engineering: C 33, 4427-4434.

Muschler, G. Chizu, N and Griffith, G. (2004). Engineering Principles of Clinical CellBased Tissue Engineering. J Bone Joint Surg Am. 86, 1541-1558.

Nieto, A., Balas, F., Colilla, M., Manzano, M and Vallet-Regí, M. (2008). Functionalization degree of SBA-15 as key factor to modulate sodium alendronate dosage.116 (1-3): 4-13.

Nieto, A., Areva, S., Wilson, T., Viitala, R., Vallet-Regí, M. (2009). Cell viability in a wet silica gel. Acta Biomaterialia. 5, 3478-3487. 
Nieto, A. (2011). Aplicaciones biomédicas de materiales mesoporosos de sílice y de carbón. Universidad complutense de Madrid: Tesis Doctoral.

Pérez, L., Lalueza, P., Monzon, M., Puertolasc, J., Arruebo, M., Santamaría, J. (2011). Hollow porous implants filled with mesoporous silica particles as a two-stage antibiotic-eluting device. International Journal of Pharmaceutics. 409, 1-8.

Peters, T. (1996). All About Albumin: Biochemistry, Genetics and Medical Applications. (Academic Press, 1st Ed, 482 pgs) San Diego, USA.

Phan, P., Grzanna, M., Chu, J., Polotsky, A., El-ghannam, A., Van Heerden, D., Hungerford, D., Frondoza, C. (2003). The effect of silica-containing calcium-phosphate particles on human osteoblasts in vitro. Journal of Biomedical Materials Research, Part A. 67 (3), 1001-1008.

Pollak, M. (2012). The insulin and insulin-like growth factor receptor family in neoplasia: an update. Nat. Rev. Cancer. 12, 159-169.

Porter, A., Botelho, C., Lopes, M., Santos, J., Best, S., Bonfield, W. (2004). Ultrastructural comparison of dissolution and apatite precipitation on hydroxyapatite and siliconsubstituted hydroxyapatite in vitro and in vivo. J Biomed Mater Res. 69, 670-9.

Qianjun, H and Jianlin, S. (2011). Mesoporous silica nanoparticle based nano drug delivery systems: synthesis, controlled drug release and delivery, pharmacokinetics and biocompatibility. Mater. Chem., 21, 5845.

Quan, Z., Han, X., Ye, Z., Chenzhong, Y., Wenjun, C. (2012). Influence of Novel NanoMesoporous Bioactive Glass on the Regulation of IGF-II Gene Expression in Osteoblasts. Cell Biochem Biophys. 62,119-123.

Quarto, M., Longaker, T. (2006). FGF-2 inhibits osteogenesis in mouse adipose tissuederived stromal cells and sustains their proliferative and osteogenic potential state, Tissue Eng. 12, 1405-1418.

Radu, C., Lai, K., Jeftinija, E., Rowe, S., Jeftinija, V and Lin. A. (2004). Polyamidoamine dendrimer-capped mesoporous silica nanosphere-based gene transfection reagent. J. Am. Chem. Soc. 126: 13216-13217. 
Raisz, L and Rodan, G. (2003). Pathogenesis of osteoporosis. Endocrinol Metab Clin North Am. 32, 15-24.

Ratner, B., Hoffman, A., Schoen, F., y Lemons, J. (2004). Biomaterials science - An introduction to materials in medicine. (2nd Ed. 879 pgs) Academic Press, segunda edición.

Ren M, Zhen H, Jinglai L, Feng G, Ouyang S. (2015). Ascorbic acid delivered by mesoporous silica nanoparticles induces the differentiation of human embryonic stem cells into cardiomyocytes. Materials Science and Engineering C. 56:348-355. DOI:

Rouquerol, D., Avnir, C., Fairbridge, W., Everett, D., Haynes, J., Pernicone, N., Ramsay, J., Sing, K and Unger. (1994). Recommendations for the characterization of porous solids. Pure \& Appl. Chern. 66 (8): 1739-1758.

Shi, X., Wang, Y., Ren, L., Zhao,N., Gong, Y., Dong-An Wang. (2009). Novel mesoporous silica-based antibiotic releasing scaffold for bone repair. Acta Biomaterialia. 5: 1697-1707.

Shi, X., Wang, Y., Rohan, R., Varshney, L., Zhang and Wang. (2009). In vitro osteogenesis of synovium stem cells induced by controlled release of bisphosphate additives from microspherical mesoporous silica composite. Biomaterials. 30: 3996-4005.

Shin-Hee, J., Eun-Jung, L., Hyoun-Ee, K., Jun-Hyeog, J., Young-Hag, K. (2011). Silica-chitosan hybrid coating on Ti for controlled release of growth factors. J Mater Sci: Mater Med. 22, 2757-2764.

Shou-Cang, S., Kiong, W., Chiaa, L., Hua, J., Tan, R. (2011). Physical state and dissolution of ibuprofen formulated by co-spray drying with mesoporous silica: Effect of pore and particle size. International Journal of Pharmaceutics. 410,188-195.

Sims, N y Gooi, J. (2008). Bone remodeling: Multiple cellular interactions required for coupling of bone formation and resorption. Seminars in Cell \& Developmental Biology. 19 (5), 444-451.

Sousa, K., Souza, E., and Sousa. (2008). Mesoporous silica/apatite nanocomposite: special synthesis route to control local drug delivery. Acta Biomater. 4671-679. 
Steven, M. M., and George, J. (2005). Exploring and Engineering the Cell Surface Interface. Science. 310, 1135-1138.

Stewart, S., Bryant, S., Ahn, J., and Hankenson. (2015). Bone Regeneration. Translational Regenerative Medicine, 313-333.

Su, J., Cao, L., Yu, B., Song, S., Liu, X., Wang, Z and Li, M. (2012). Composite scaffolds of mesoporous bioactive glass and polyamide for bone repair. International Journal of Nanomedicine. 7, 2547-2555.

Su, W., Wei, T., Lu, M., Meng, Z., Chen, X., Jing, J Fu, T. (2019). Treatment of metastatic lung cancer via inhalation administration of curcumin composite particles based on mesoporous silica. European Journal of Pharmaceutical Sciences, 134, 246255

Sun, J., Wei, L., Liu, X et al. (2009). Acta Biomater. 5, 1284.

Tang, Q., Xu, Y., Wu, D and Sun, Y.(2006). A study of carboxylic-modified mesoporous silica in controlled delivery for drug famotidine. J. Solid State Chem. 179,15131520.

Thielemann, F. Girgsdies, R. Schlögl, C. Hess, Beilstein J. (2011). Pore structure and surface area of silica SBA-15: influence of washing and scale-up. Nanotechnol. 2, 110-118.

Ukmar, T and Planinsek, O. (2010). Ordered mesoporous silicates as matrices for controlled release of drugs. Acta Pharm. 60: 373-385.

Van de Manakker, F., Braeckmans, K., El Morabit, N., De Smedt, S., Van Nostrum, C., and Hennink, W. (2009). Protein-release behavior of self-assembled PEG-betacyclodextrin/PEG-cholesterol hydrogels. Adv. Func. Mater. 19, 2992-3001.

Vega, J., lyoshia, M., Kima, K., Hozumib, A., Sugimuraa, H and Takai, O. (2001). Spin casted mesoporous silica coatings for medical applications. Thin Solid Films. 398 $-399,615-620$. 
Vivero-Escoto, J. (2009). Surface functionalized mesoporous silica nanoparticles for intracellular drug delivery. (lowa St Univ. PhD thesis, 160 pgs) 10930.

Wang, T., Chai, F., Wang, C., Li, L., Hai-Yan, L., Zang, L., Zhong-Min, S and Liao, Y. (2011). Fluorescent hollow/rattle-type mesoporous Au@SiO2 nanocapsules for drug delivery and fluorescence imaging of cancer cells. Journal of Colloid and Interface Science. 358, 109-115.

Wang, Y., Zhao, Q. F., Han, N., Bai, L., Li, J., Liu, J., Che, E. X., Hu, L., Zhang, Q., Jiang, T. Y., Wang, S. L. (2015). Mesoporous silica nanoparticles in drug delivery and biomedical applications. Nanomed.-Nanotechnol. Biol. Med. 11 (2), 313-327.

Wei, G., Jin, Q., Giannobile, W., Ma, P. (2007). The enhancement of osteogenesis by nano-fibrous scaffolds incorporating rhBMP-7 nanospheres. Biomaterials. 28: 2087-2096.

Williams D.F. (2008). On the mechanisms of biocompatibility. Biomaterials, 29, 29412953

Wu, J., Kusuma, C., Mond, J., Kokai-Kun, J. (2003). Antimicrob. Agents Chemother. 47, 3407-3414.

Wu, C., Fan, W., Gelinsky, M., Xiao, Y., Chang, J., Thor, F and Gianaurelio. (2011). In situ preparation and protein delivery of silicate-alginate composite microspheres with core-shell structure. J. R. Soc. Interface. 8, 1804-1814.

Wozney, J., Rosen, V., Celeste, L., Mitsock, M., M.J. Whitters, M., Kriz, R., Hewick, E., and Wan. (1988). Novel regulators of bone formation: molecular clones and activities. Science. 242: 1528-1534.

Yachao, J., Zhang, P., Sun, Y., Kang, Q., Xu, J., Zhang, C., \& Chai, Y. (2019). Regeneration of large bone defects using mesoporous silica coated magnetic nanoparticles during distraction osteogenesis. Nanomedicine: Nanotechnology, Biology and Medicine, 102040.

Yanagisawa, T., Shimizu, T., Kuroda, K and Kato, C. (1990). The Preparation of Alkyltrimethylammonium-Kanemite Complexes and Their Conversion to Microporous Materials. Bull.Chem.Soc. Jpm. 63 (4): 988-992. 
Ying, J., Mehnert, C., Wong, M., Angew, S. (1999). Chem. Int. 38: 56-77.

Yilgor, P., Hasirci, N., Hasirci, V. (2009). Sequential BMP-2/BMP-7 delivery from polyester nanocapsules. Journal of Biomedical Materials Research, Part A, 528-536.

Yilgor, P., Tuzlakoglu, K., Reis, R., Hasirci, N., Hasirci, V. (2009). Incorporation of a sequential BMP-2/BMP-7 delivery system into chitosan-based scaffolds for bone tissue engineering. Biomaterials. 30, 3551-3559.

Yiu, H., Wright, P. (2005). Mater. Chem. 15, 3690-3700.

Zanjanizadeh E., Shahbazi, M., Shatalin, V., Nadal, E., Mäkilä, E., Salonen, J., Kemell, M., Correia, A., Hirvonen, J and Santos. (2018). Conductive vancomycin-loaded mesoporous silica polypyrrole-based scaffolds for bone regeneration. International Journal of Pharmaceutics, 536(1), 241-250.

Zeid, A. (2012). Fundamental Aspects of Silicate Mesoporous Materials. Materials, 5, 2874-2902.

Zhao D, Feng J, Qisheng H, Melosh N, Fredrickson G H, Chmelka B F and Stucky G D. (1998). Triblock Copolymer Syntheses of Mesoporous Silica with Periodic 50 to 300 Angstrom Pores. Science 279(5350), 548-552.

Zhao, Y., Trewyn, B., Slowing, I and Lin, V. (2009). Mesoporous silica nanoparticlebased double drug delivery system for glucose-responsive controlled release of insulin and cyclic AMP. J. Am. Chem.Soc. 131: 8398-8400. 